\title{
Qualitative Properties of the Solution to Brinkman-Stokes System Modelling a Filtration Process
}

\author{
Yulia Koroleva
}

Department of Higher Mathematics, Gubkin State University of Oil and Gas, (National Research University), Russia

Copyright (C) 2017 by authors, all rights reserved. Authors agree that this article remains permanently open access under the terms of the Creative Commons Attribution License 4.0 International License

\begin{abstract}
The paper deals with study of Stokes-Brinkman system with varying viscosity that describes the fluid flow along an ensemble of partially porous cylindrical particles using the cell approach. Existence and uniqueness of the solution to the system is proved for an arbitrary varying viscosity. Some uniform estimates on the velocity of flow are derived. Moreover, an axillary weighted Friedrichs inequality was proved for the solution of the considered system. The numerical illustration of the obtained results is given.
\end{abstract}

Keywords Fluid Flow, Stokes System, Brinkman's Equation, Varying Viscosity

\section{Introduction}

The development of modern nano-technologies requires the knowledge of filtration processes. They can be modelled as flow through porous medium. Some important examples of flow through porous medium are pressure driven membrane processes, flows of underground water and crude oil. Usually porous medium is modeled by a dense set of rigid impermeable particles [1]. However, the structure of a porous layer should be viewed from different points of view. For example, it is not necessary that the particles always have a smooth homogeneous surface, they can have a rough surface or a surface covered by a porous shell. The cell model [1] has been effectively used for studying of the mentioned above flows. The basic principle of the cell model is to replace the system of randomly oriented particles by a periodic array of spheres or cylinders embedded in the centrally spherical or centrally cylindrical liquid cells. Boundary conditions on the cell boundary must describe the influence of surrounding particles on the flow inside the cell and the force acting to the particle in the center of the cell. There are four types of these conditions: the Happel (the absence of tangential stresses on the cell surface), Kuwabara (the absence of vortexes, equivalently, the flow potentiality), Kvashnin (the cell symmetry), and Cunningham (the flow on the surface of cell is assumed to be uniform) models [2].

Flow through porous shells is frequently modeled by Brinkmans equation [4]. However, it has been observed that the obtained results based on the Brinkmans equations do not agree with the experimental data for non-homogeneous porous media. For that reason a modification of the Brinkmans equation was suggested in [14] for the media having non-homogeneous porosity. The possible way to overcome this problem it to use variable viscosity model for the liquid/porous boundary region. We assume that considered porous shells have a uniform porosity but variable liquid viscosity inside porous layer. It is supposed that the investigated membranes are built by either non-porous particles with rough surface or particles covered by porous shell. The latter shells have rough surface and the scale of roughness is equal to or bigger than the average pore size inside the shell. The important problem is to select correctly boundary conditions. We use bellow the condition of "tangential stresses slippage", i.e. the presence of tangential stresses jump at the porous-liquid interface [8],[9].

In present paper the described physical problem was stated strongly mathematically. The unknown velocity of flow was considered as the weak solution of the Brinkman-Stokes system. The aim is to investigate qualitative properties of the considered model. We prove the existence and uniqueness of the weak solution as well as derive uniform estimates which show the behaviour of the flow depending on the variable viscosity. Let us mention that similar problems for constant viscosity and spherical particles were solved analytically in [10] and [11]. The case of flow with varying viscosity can not be treated analytically in general, perhaps except some particular situations. Therefore the fact of existence and uniqueness of the solution is the important result. Moreover, the 
derived estimates give a restriction on growth of the solution and confirm the natural phenomena: the greater viscosity the less the speed of the flow. In the analysis we have derived an independently important fact on validity of weighted Friedrich's inequality.

The obtained results are validated numerically. The visualisation for graphs of the solution to Stokes-Brinkman system is presented in Section 4 for the case of polynomial viscosity.

\section{Statement of the problem}

The viscous flow through porous medium modelled as a set of parallel composite cylindrical particles and filled by liquid with varying viscosity can be described by two systems: the Stokes one

$$
\left\{\begin{array}{l}
\widetilde{\nabla} \widetilde{p}^{o}=\widetilde{\mu}^{0} \widetilde{\Delta} \widetilde{\mathbf{v}}^{0} \\
\widetilde{\operatorname{div}} \widetilde{\mathbf{v}}^{o}=0
\end{array}\right.
$$

outside the porous layer $\widetilde{a} \leq \widetilde{r} \leq \widetilde{b}$ and $\widetilde{R} \leq \widetilde{r} \leq \widetilde{a}$ by the Brinkman's system

$$
\left\{\begin{array}{l}
\widetilde{\nabla} \widetilde{p}^{i}=\widetilde{\operatorname{div}}\left(\widetilde{\mu}^{i} \widetilde{D} \widetilde{\mathbf{v}}^{i}\right)-\frac{\widetilde{\mu}^{o}}{\widetilde{k}} \widetilde{\mathbf{v}}^{i} \\
\widetilde{\operatorname{div}} \widetilde{\mathbf{v}}^{i}=0
\end{array}\right.
$$

in the porous layer. Here the tilde denotes dimensional variables, indices $o$ and $i$ refer to the external and porous zones respectively; $\widetilde{\mu}^{i}$ and $\widetilde{\mu}^{o}$ are the viscosities inside of Brinkman's layer and outside of it, correspondingly. The variable $\widetilde{k}$ is the specific permeability of the porous layer. We suppose that viscosity of clear liquid $\widetilde{\mu}^{o}$ is constant over region $\widetilde{a}<\tilde{r}<\tilde{b}$ while the viscosity of Brinkmans liquid $\tilde{\mu}^{i}=\tilde{\mu}^{o} \mu(r), \mu(r)>0$ is the function of $r$ variable. In general case the unknown functions are velocities $\widetilde{\mathbf{v}}^{o}, \widetilde{\mathbf{v}}^{i}$ and pressures $\widetilde{p}^{o}, \widetilde{p}^{i}$ of flow. lid:

The following boundary conditions are assumed to be va-

$$
\begin{gathered}
\widetilde{\mathbf{v}}^{i}=0, \text { as } \widetilde{r}=\widetilde{R}, \\
\widetilde{\mathbf{v}}^{i}=\widetilde{\mathbf{v}}^{o}, \quad \widetilde{\sigma}_{r r}^{o}=\widetilde{\sigma}_{r r}^{i}, \text { as } \widetilde{r}=\widetilde{a} .
\end{gathered}
$$

Here (4) is the continuity condition. The assumption for the jump of tangential stresses at the interface between porous layer and clear liquid reads

$$
\widetilde{\sigma}_{r z}^{i}-\widetilde{\sigma}_{r z}^{o}=\frac{\widetilde{\beta \mu^{o}}}{\sqrt{\tilde{k}}} \widetilde{v}_{z}^{o}, \text { as } \widetilde{r}=\widetilde{a} .
$$

Here $-\infty<\beta<\sup _{r} \sqrt{\frac{\tilde{\mu}^{i}}{\tilde{\mu}^{0}}}$ is the dimensionless parameter which can be found from a physical experiment [5]. In case when flow is parallel to the cylinders all four known conditions at the outer cell boundary are reduced to the scalar one [12]:

$$
\frac{d \widetilde{v}_{z}^{o}}{d \widetilde{r}}=0, \text { as } \widetilde{r}=\widetilde{b}
$$

Let us pass to the dimensionless operators and variables by the following substitutions:

$$
\begin{aligned}
& \frac{\tilde{b}}{\tilde{a}}=\frac{1}{\gamma}, r=\frac{\tilde{r}}{\tilde{a}}, z=\frac{\tilde{z}}{\tilde{a}}, \nabla=\tilde{\nabla} \cdot \tilde{a}, \Delta=\tilde{\Delta} \cdot \tilde{a}^{2}, \\
& \delta=\frac{\tilde{\delta}}{\tilde{a}}, R=\frac{\tilde{R}}{\tilde{a}}=1-\delta, \mathbf{v}=\frac{\tilde{\mathbf{v}}}{\tilde{U}} \quad p=\frac{\tilde{p}}{\tilde{p}_{0}} \\
& \tilde{p}_{0}=\frac{\tilde{U} \cdot \tilde{\mu}^{o}}{\tilde{a}}, k=\frac{\tilde{k}}{\tilde{a}^{2}}>0, \omega=\frac{d p}{d z} .
\end{aligned}
$$

Here $\widetilde{U}$ is the cell (filtration) velocity $\tilde{U}=-\tilde{L}_{11} \frac{d \tilde{p}}{d \tilde{z}}$, where $\tilde{L}_{11}$ is the hydrodynamic permeability of the membrane [13], and $\omega<0$ is a given pressure gradient.

Denote by $B_{\gamma}$ the layer

$$
B_{\gamma}=\left\{1 \leq r \leq \frac{1}{\gamma}, \varphi \in[0,2 \pi], z \in[0, \infty)\right\}
$$

and by $B_{R}$ the set

$$
B_{R}=\{R \leq r \leq 1, \varphi \in[0,2 \pi], z \in[0, \infty)\} .
$$

In the dimensionless notations the systems (1) and (2) read as

$$
\left\{\begin{array}{l}
\nabla p^{o}=\Delta \mathbf{v}^{o} \text { in } B_{\gamma} \\
\operatorname{div} \mathbf{v}^{o}=0 \text { in } B_{\gamma} \\
\frac{d v_{z}^{o}}{d r}=0 \text { on } r=\frac{1}{\gamma}, v_{z}^{o}=v_{z}^{i} \text { on } r=1
\end{array}\right.
$$

and

$$
\left\{\begin{array}{l}
\nabla p^{i}=\operatorname{div}\left(\mu(r) D \mathbf{v}^{i}\right)-\frac{\mathbf{v}^{\mathbf{i}}}{k} \text { in } B_{R} \\
\operatorname{div} \mathbf{v}^{i}=0 \text { in } B_{R} \\
v_{z}^{i}=0 \text { on } r=R \\
\frac{d v_{z}^{i}}{d r}=\frac{d v_{z}^{o}}{d r}+\frac{\beta}{\sqrt{k}} v_{z}^{o} \text { as } r=1 .
\end{array}\right.
$$

Problems (8) and (9) are linked to each other via the boundary conditions on the common boundary $r=1$ which physically means the continuous flow regime.

Our goal is to investigate the qualitative properties of the obtained systems. It is necessary to verify rigorously the existence and uniqueness of the solution as well as to derive apriori estimates which can be useful to understand the behaviour of flow.

\subsection{The flow parallel to cylinders.}

Let us rewrite the problems (8) and (9) in cylindric coordinates $(r, \varphi, z)$ with help of formulas

$$
\begin{aligned}
& \nabla p=\left(\frac{\partial p}{\partial r}, \frac{1}{r} \frac{\partial p}{\partial \varphi}, \frac{\partial p}{\partial z}\right) \\
& \operatorname{div} v=\left(\frac{1}{r} \frac{\partial}{\partial r}\left(r v_{r}\right)+\frac{1}{r} \frac{\partial v_{\varphi}}{\partial \varphi}+\frac{\partial v_{z}}{\partial z}\right), \\
& \Delta v=\frac{1}{r} \frac{\partial}{\partial r}(r v)+\frac{1}{r^{2}} \frac{\partial^{2} v}{\partial \varphi^{2}}+\frac{\partial^{2} v}{\partial z^{2}}
\end{aligned}
$$


We shall consider the case of flow parallel to cylinders. Under this assumption, the components of the solution satisfy $v_{r}^{i}=v_{r}^{o}=v_{\varphi}^{i}=v_{\varphi}^{o}=0$, while $v_{z}^{i} \neq 0, v_{z}^{o} \neq 0$. It can be shown that velocity and pressure derivative $\frac{\partial p^{j}}{\partial z}, j=o, i$ are independent on $z$ - variable. Indeed, for $j=o$ or $j=i$ the equation

$\operatorname{div} \mathbf{v}^{j}=0 \Leftrightarrow\left(\frac{1}{r} \frac{\partial}{\partial r}\left(r v_{r}^{j}\right)+\frac{1}{r} \frac{\partial v_{\varphi}^{j}}{\partial \varphi}+\frac{\partial v_{z}^{j}}{\partial z}\right)=0 \Leftrightarrow \frac{\partial v_{z}^{j}}{\partial z}=0$

implies independence $v_{z}^{j}$ on $z$ - variable. For an arbitrary $\mu^{i}(r)$ when the flow is parallel to z-direction, the term $\operatorname{div}\left(\mu^{i} D \mathbf{v}\right)$ becomes

$$
\frac{1}{r} \frac{d}{d r}\left(\mu^{i}\left(r \frac{d v_{z}}{d r}\right)\right)=\frac{d v_{z}}{d r}\left(\frac{d \mu^{i}}{d r}+\frac{\mu^{i}}{r}\right)+\mu^{i} \frac{d^{2} v_{z}}{d r^{2}}
$$

in the polar coordinates. Having in mind that

$$
\frac{d p^{j}}{d z}=\text { const }=\omega(<0), j=o, i,
$$

we arrive at the following one-dimensional Stokes and Brinkman's equations where for the simplicity we omit the subindex $z$ (i.e. the notation $v^{j}$ should be understood as $v_{z}^{j}$ ):

$$
\begin{gathered}
\frac{d^{2} v^{o}}{d r^{2}}+\frac{1}{r} \frac{d v^{o}}{d r}=\omega, 1<r<\frac{1}{\gamma}, \\
\mu \frac{d^{2} v^{i}}{d r^{2}}+\left(\frac{d \mu}{d r}+\frac{\mu}{r}\right) \frac{d v^{i}}{d r}=\left(\frac{v^{i}}{k}+\omega\right), R<r<1
\end{gathered}
$$

with boundary conditions

$$
\begin{aligned}
& v^{i}=0 \text { as } r=R, \\
& v^{o}=v^{i} \text { as } r=1, \quad \frac{d v^{i}}{d r}-\frac{d v^{o}}{d r}=\frac{\beta}{\sqrt{k}} v^{o}, \quad r=1 . \\
& \frac{d v^{o}}{d r}=0 \text { as } r=\frac{1}{\gamma} .
\end{aligned}
$$

\section{On the existence and uniqueness of the solution.}

\subsection{Preliminaries.}

In our analysis the following classical theorem will be used (see [3] and [6]):

Theorem 1 (Lions-Lax-Milgram Lemma). Let $U$ and $V$ be two real Hilbert spaces and let

$$
B: U \times V \rightarrow \mathbb{R}
$$

be a continuous bilinear functional where $V$ is continuously embedded in $U\left(\|u\|_{U} \leq c\|u\|_{V}\right)$. Suppose also that $B$ is coercive in the following sense: for some constant $c>0$ and all $u \in U,|B[u, u]| \geq c\|u\|_{U}^{2}$. Then for all $f \in V^{*}$ there exists the unique solution $u=u_{f} \in U$ to weak problem $B\left[u_{f}, v\right]=\langle f, v\rangle$ for all $v \in V$. Moreover, the solution depends continuously on the given datum: $\left\|u_{f}\right\|_{U} \leq \frac{1}{c}\|f\|_{V^{*}}$.
Let $H^{1}(\Omega)$ be the Sobolev space defined as the completion of the set of functions from space $C^{\infty}(\bar{\Omega})$ by the norm

$$
\|u\|_{H^{1}(\Omega)}=\sqrt{\int_{\Omega}\left(u^{2}+|\nabla u|^{2}\right) d x} ;
$$

$L_{1}(\Omega)$ is the set of all measurable functions integrable in $\Omega$. Let us prove now the following important Lemma which states a weighted Friedrich's inequality. We refer to [15] for more results on Friedrich's-type inequalities in different domains.

Lemma 3.1. Assume that

$$
\mu(r)>0 \text { and } \frac{1}{\mu}, \mu \in L_{1}(R, 1) .
$$

Then the following weighted Friedrichs inequality holds for $v^{i} \in H^{1}(R, 1)$ satisfying $v^{i}(R)=0$ :

$$
\int_{R}^{1} \mu\left(v^{i}\right)^{2} d r \leq\|\mu\|_{L_{1}(R, 1)}\left\|\frac{1}{\mu}\right\|_{L_{1}(R, 1)} \int_{R}^{1} \mu\left(\frac{d v^{i}}{d r}\right)^{2} d r .
$$

Proof. Since $v^{i}(R)=0$, it holds that

$$
v^{i}(r)=v^{i}(r)-v^{i}(R)=\int_{R}^{r} \frac{d v^{i}}{d r} d r
$$

and by Hölder inequality

$$
\left(v^{i}\right)^{2}=\left(\int_{R}^{r} \frac{\sqrt{\mu}}{\sqrt{\mu}} \frac{d v^{i}}{d r} d r\right)^{2} \leq \int_{R}^{1} \frac{1}{\mu} d r \int_{R}^{1} \mu\left(\frac{d v^{i}}{d r}\right)^{2} d r .
$$

Hence,

$$
\mu(r)\left(v^{i}\right)^{2} \leq \mu(r) \int_{R}^{1} \frac{1}{\mu} d r \int_{R}^{1} \mu\left(\frac{d v^{i}}{d r}\right)^{2} d r .
$$

Integrating once more, we obtain

$$
\begin{aligned}
& \int_{R}^{1} \mu\left(v^{i}\right)^{2} d r \leq \int_{R}^{1} \frac{1}{\mu} d r \int_{R}^{1} \mu d r \int_{R}^{1} \mu\left(\frac{d v^{i}}{d r}\right)^{2} d r= \\
& \|\mu\|_{L_{1}(R, 1)}\left\|\frac{1}{\mu}\right\|_{L_{1}(R, 1)} \int_{R}^{1} \mu\left(\frac{d v^{i}}{d r}\right)^{2} d r .
\end{aligned}
$$

\subsection{The weak solution}

Multiplying equations (11), (12) by $v^{o}, v^{i}$ respectively and integrating the result over the corresponding domains, we can define the weak solutions $v^{o}$ and $v^{i}$. 
Definition 1. The function $v^{o} \in H^{1}\left(B_{R}\right)$ is called the weak solution to (11) if the following integral identity holds:

$-\int_{1}^{\frac{1}{\gamma}}\left(\frac{d v^{o}}{d r}\right)^{2} d r+\int_{1}^{\frac{1}{\gamma}} \frac{v^{o}}{r} \frac{d v^{o}}{d r} d r=\left.\frac{d v^{o}}{d r}\right|_{r=1} v^{o}(1)+\omega \int_{1}^{\frac{1}{\gamma}} v^{o} d r$

The function $v^{i} \in H^{1}\left(B_{R}\right)$ is called the weak solution to (12) if it satisfies

$$
\begin{aligned}
& \int_{R}^{1} \mu v^{i} \frac{d^{2} v^{i}}{d r^{2}} d r+\int_{R}^{1}\left(\frac{d \mu}{d r}+\frac{\mu}{r}\right) v^{i} \frac{d v^{i}}{d r} d r-\frac{1}{k} \int_{R}^{1}\left(v^{i}\right)^{2} d r= \\
& \omega \int_{R}^{1} v^{i} d r .
\end{aligned}
$$

Using integration by parts and boundary conditions for $v^{i}$ it is possible to rewrite (16) to the form

$$
\begin{aligned}
& \int_{R}^{1} \mu\left(\frac{d v^{i}}{d r}\right)^{2} d r-\int_{R}^{1} \frac{\mu}{r} v^{i} \frac{d v^{i}}{d r} d r+\frac{1}{k} \int_{R}^{1}\left(v^{i}\right)^{2} d r= \\
& \left.\mu(1) v^{i}(1) \frac{d\left(v^{i}\right)}{d r}\right|_{r=1}-\omega \int_{R}^{1} v^{i} d r .
\end{aligned}
$$

\subsection{The main result}

Let us examine solvability of proposed problems. The following theorems give such result.

Theorem 2. The function

$$
v^{o}=C-\frac{\omega}{2 \gamma^{2}} \ln r+\frac{\omega r^{2}}{4}, \quad \text { where } C=v^{i}(1)-\frac{\omega}{4}
$$

is the unique solution to Stokes problem in the layer $B_{\gamma}$.

Proof. It is easy to find the analytical solution to (11), which evidently coincides with the solution (15) in the weak sense. Indeed,

$$
\frac{d}{d r}\left(r \frac{d v^{o}}{d r}\right)=r \omega \Leftrightarrow \frac{d v^{o}}{d r}=\frac{r \omega}{2}+\frac{C}{r} .
$$

Boundary condition $\frac{d v^{o}}{d r}=0$ at $r=\frac{1}{\gamma}$ implies that

$$
C=-\frac{\omega}{2 \gamma^{2}} .
$$

Integrating the equation once more and taking into the account condition $v^{o}=v^{i}$ at $r=1$, one derives (18) The uniqueness of $v^{o}$ follows directly from formula (18) as soon as $v^{i}$ is the uniquely defined (see Theorem 3).
Theorem 3. Consider $\mu(r)>0$ such that

$$
\frac{1}{\mu}, \mu \in L_{1}(R, 1) \text {. }
$$

There exists the unique weak solution $v^{i} \in H^{1}\left(B_{R}\right)$ to (17) which satisfies estimates

$$
\begin{aligned}
&\left\|\sqrt{\mu} v^{i}\right\|_{L_{2}(R, 1)}^{2} \leq C_{1}\left(\frac{\beta}{\sqrt{k}} \mu(1)\left(v^{o}(1)\right)^{2}+\right. \\
&\left.\frac{\mu(1) v^{o}(1)(-\omega)}{2}\left(\frac{1}{\gamma^{2}}-1\right)\right), \\
&\left\|\sqrt{\mu} \frac{d v^{i}}{d r}\right\|_{L_{2}(R, 1)}^{2} \leq C_{2}\left(\frac{\beta}{\sqrt{k}} \mu(1)\left(v^{o}(1)\right)^{2}+\right. \\
&\left.\frac{\mu(1) v^{o}(1)(-\omega)}{2}\left(\frac{1}{\gamma^{2}}-1\right)\right),
\end{aligned}
$$

$$
\begin{aligned}
& \text { if } \beta \geq 0 \text { and } \\
& \left\|\sqrt{\mu} v^{i}\right\|_{L_{2}(R, 1)}^{2} \leq C_{1} \frac{\mu(1) v^{o}(1)(-\omega)}{2}\left(\frac{1}{\gamma^{2}}-1\right), \\
& \left\|\sqrt{\mu} \frac{d v^{i}}{d r}\right\|_{L_{2}(R, 1)}^{2} \leq C_{2} \frac{\mu(1) v^{o}(1)(-\omega)}{2}\left(\frac{1}{\gamma^{2}}-1\right), \\
& \text { if } \beta<0,
\end{aligned}
$$

where $v^{o}$ is given by (18). Here

$C_{1}=\left(\left(\omega+\frac{1}{k}\right) \frac{1}{\sup _{(R, 1)} \mu}-\frac{1}{2 \sigma}\right)^{-1}$,

$C_{2}=\left(1-\frac{\sigma}{2 R^{2}}\right)^{-1}$,

if $\frac{1}{2(\omega k+1)} k \sup _{(R, 1)} \mu<2 R^{2}$;

$C_{1}=\frac{\|\mu\|_{L_{1}(R, 1)}\left\|\frac{1}{\mu}\right\|_{L_{1}(R, 1)}}{\left(\frac{3}{4}-\|\mu\|_{L_{1}(R, 1)}\left\|\frac{1}{\mu}\right\|_{L_{1}(R, 1)}\left(\frac{1}{R^{2}}-\frac{k \omega+1}{k} \frac{1}{\sup _{(R, 1)} \mu}\right)\right)}$,

$C_{2}=\left(\frac{3}{4}-\|\mu\|_{L_{1}(R, 1)}\left\|\frac{1}{\mu}\right\|_{L_{1}(R, 1)}\left(\frac{1}{R^{2}}-\frac{k \omega+1}{k} \frac{1}{\sup _{(R, 1)} \mu}\right)\right)^{-1}$,

if $\frac{1}{2(\omega k+1)} k \sup _{(R, 1)} \mu \geq 2 R^{2}$

for any positive constant $\sigma$ such that

$$
\frac{1}{2(\omega k+1)} k \sup _{(R, 1)} \mu<\sigma<2 R^{2} .
$$

Proof. We shall establish now the existence and uniqueness of the solution $v^{i}$. Denote by

$$
B\left[v^{i}, v^{i}\right]: H^{1}\left(B_{R}\right) \times H^{1}\left(B_{R}\right) \rightarrow \mathbb{R}
$$


the bilinear form

$B\left[v^{i}, v^{i}\right]=\int_{R}^{1} \mu\left(\frac{d v^{i}}{d r}\right)^{2} d r-\int_{R}^{1} \frac{\mu}{r} v^{i} \frac{d v^{i}}{d r} d r+\frac{1}{k} \int_{R}^{1}\left(v^{i}\right)^{2} d r$.

Define the following functional on space $H^{1}\left(B_{R}\right)$ :

$$
\begin{gathered}
\left\langle f, v^{i}\right\rangle=-v^{i}(1) C-\omega \int_{R}^{1} v^{i} d r, \\
C=\frac{\mu(1) \omega}{2}\left(1-\frac{1}{\gamma^{2}}\right)+\frac{\mu(1) \beta}{\sqrt{k}} v^{o}(1) .
\end{gathered}
$$

Thus, the question on existence and uniqueness of the weak solution (17) is reduced to solvability of

$$
B\left[v^{i}, v^{i}\right]=\left\langle f, v^{i}\right\rangle
$$

for any $f \in H^{-1}\left(B_{R}\right)$. Let us establish the coerciveness of form $B\left[v^{i}, v^{i}\right]$ (see Theorem 1).

In order to do that, we proceed with derivation of auxiliary inequalities.

Since $\sup _{r} \mu \neq 0$, it holds that

$$
\begin{gathered}
\frac{1}{k} \int_{R}^{1}\left(v^{i}\right)^{2} d r \geq \frac{1}{k \sup _{(R, 1)} \mu} \int_{R}^{1} \mu\left(v^{i}\right)^{2} d r, \\
-\omega \int_{R}^{1} v^{i} d r \leq \frac{-\omega}{\sup _{(R, 1)} \mu} \int_{R}^{1} \mu\left(v^{i}\right)^{2} d r .
\end{gathered}
$$

Next,

$$
\begin{gathered}
a b \leq \frac{\sigma a^{2}}{2}+\frac{b^{2}}{2 \sigma} \text { for any } \sigma>0 \Longrightarrow \\
\text { for } a=\sqrt{\mu} \frac{v^{i}}{r}, b=\sqrt{\mu} \frac{d v^{i}}{d r}
\end{gathered}
$$

one gets

$$
\begin{aligned}
& -\int_{R}^{1} \frac{\mu}{r} v^{i} \frac{d v^{i}}{d r} d r \geq-\frac{\sigma}{2} \int_{R}^{1}\left(\sqrt{\mu} \frac{v^{i}}{r}\right)^{2} d r \\
& -\frac{1}{2 \sigma} \int_{R}^{1}\left(\sqrt{\mu} \frac{d v^{i}}{d r}\right)^{2} d r \\
& \geq-\frac{\sigma}{2 R^{2}} \int_{R}^{1}\left(\sqrt{\mu} v^{i}\right)^{2} d r-\frac{1}{2 \sigma} \int_{R}^{1}\left(\sqrt{\mu} \frac{d v^{i}}{d r}\right)^{2} d r .
\end{aligned}
$$

Applying inequalities (20), (22) with e.g. $\sigma=R^{2}$, we conclude that

$$
\begin{aligned}
& \left|B\left[v^{i}, v^{i}\right]\right| \geq \mid\left(1-\frac{1}{2 R^{2}}\right) \int_{R}^{1}\left(\sqrt{\mu} \frac{d v}{d r}\right. \\
& \left(\frac{1}{k \sup _{(R, 1)} \mu}-\frac{1}{2}\right) \int_{R}^{1}\left(\sqrt{\mu} v^{i}\right)^{2} d r \mid \geq \\
& \inf _{(R, 1)}\left|1-\frac{1}{2 R^{2}}\right| \int_{R}^{1}\left(\frac{d v^{i}}{d r}\right)^{2} d r+ \\
& \inf \mu\left|\frac{1}{k \sup _{(R, 1)}}-\frac{1}{2}\right| \int_{R}^{1}\left(v^{i}\right)^{2} d r \geq \\
& C(\mu)\left\|v^{i}\right\|_{H_{1}\left(B_{R}\right)}^{2},
\end{aligned}
$$

where

$$
C(\mu)=\inf _{(R, 1)} \mu \min \left\{\left|1-\frac{1}{2 R^{2}}\right|,\left|\frac{1}{k \sup _{(R, 1)} \mu}-\frac{1}{2}\right|\right\}
$$

and

$$
\left\|v^{i}\right\|_{H_{1}\left(B_{R}\right)}^{2}=\int_{R}^{1}\left(\frac{d v^{i}}{d r}\right)^{2} d r+\int_{R}^{1}\left(v^{i}\right)^{2} d r
$$

Hence, the unique weak solution $v^{i}$ exists due to Lions-LaxMilgram Lemma.

Now we derive estimates (19). Evaluating the term

$$
\left.\mu(1) v^{i}(1) \frac{d v^{i}}{d r}\right|_{r=1}
$$

one obtains

$$
\begin{aligned}
& \left.\mu(1) v^{i}(1) \frac{d v^{i}}{d r}\right|_{r=1}=v^{i}(1) C, \text { where } \\
& C=\frac{\mu(1) \omega}{2}\left(1-\frac{1}{\gamma^{2}}\right)+\frac{\mu(1) \beta}{\sqrt{k}} v^{o}(1),
\end{aligned}
$$

therefore(17) can be written as

$$
\begin{aligned}
& \int_{R}^{1} \mu\left(\frac{d v^{i}}{d r}\right)^{2} d r-\int_{R}^{1} \frac{\mu}{r} v^{i} \frac{d v^{i}}{d r} d r+\frac{1}{k} \int_{R}^{1}\left(v^{i}\right)^{2} d r= \\
& -\omega \int_{R}^{1} v^{i} d r-\frac{\mu(1) v^{o}(1) \omega}{2}\left(\frac{1}{\gamma^{2}}-1\right)+\frac{\mu(1) \beta}{\sqrt{k}}\left(v^{o}(1)\right)^{2} .
\end{aligned}
$$


Applying inequalities (20) - (22), one derives that

$$
\begin{aligned}
& \left(1-\frac{\sigma}{2 R^{2}}\right) \int_{R}^{1}\left(\sqrt{\mu} \frac{d v^{i}}{d r}\right)^{2} d r+ \\
& \left(\frac{1}{k \sup _{(R, 1)}}-\frac{1}{2 \sigma}\right) \int_{R}^{1}\left(\sqrt{\mu} v^{i}\right)^{2} d r \leq \\
& \int_{R}^{1} \mu\left(\frac{d v^{i}}{d r}\right)^{2} d r-\int_{R}^{1} \frac{\mu}{r} v^{i} \frac{d v^{i}}{d r} d r+\frac{1}{k} \int_{R}^{1}\left(v^{i}\right)^{2} d r= \\
& -\omega \int_{R}^{1} v^{i} d r-\frac{\mu(1) v^{o}(1) \omega}{2}\left(\frac{1}{\gamma^{2}}-1\right)+ \\
& \frac{\beta \mu(1)}{\sqrt{k}}\left(v^{o}(1)\right)^{2} \leq \frac{-\omega}{\sup _{(R, 1)} \mu} \int_{R}^{1} \mu\left(v^{i}\right)^{2} d r- \\
& \frac{\mu(1) v^{o}(1) \omega}{2}\left(\frac{1}{\gamma^{2}}-1\right)+\frac{\beta \mu(1)}{\sqrt{k}}\left(v^{o}(1)\right)^{2} .
\end{aligned}
$$

Thus, we can conclude that

$$
\begin{aligned}
& \left(1-\frac{\sigma}{2 R^{2}}\right) \int_{R}^{1}\left(\sqrt{\mu} \frac{d v^{i}}{d r}\right)^{2} d r+ \\
& \left(\left(\omega+\frac{1}{k}\right) \frac{1}{\sup _{(R, 1)} \mu}-\frac{1}{2 \sigma}\right) \int_{R}^{1}\left(\sqrt{\mu} v^{i}\right)^{2} d r \leq \\
& \frac{\beta \mu(1)}{\sqrt{k}}\left(v^{o}(1)\right)^{2}-\frac{\mu(1) v^{o}(1) \omega}{2}\left(\frac{1}{\gamma^{2}}-1\right) .
\end{aligned}
$$

Now we choose the constant $\sigma$ such that $\sigma<2 R^{2}$. With this choice the coefficient in front of the first term is positive. If it holds that $\frac{k}{2(\omega k+1)} \sup _{(R, 1)} \mu<2 R^{2}$ (equivalently, the second term is positive as well), then one should fix $\sigma$ satisfying $\frac{k}{2(\omega k+1)} \sup _{(R, 1)} \mu<\sigma<2 R^{2}$. If $\beta<0$, then

$$
-\frac{\beta \mu(1)}{\sqrt{k}}\left(v^{o}(1)\right)^{2}>0
$$

and it can be added to the right-hand side of (25). Thus,the estimate

$$
\begin{aligned}
& \left(1-\frac{\sigma}{2 R^{2}}\right) \int_{R}^{1}\left(\sqrt{\mu} \frac{d v^{i}}{d r}\right)^{2} d r+ \\
& \left(\left(\omega+\frac{1}{k}\right) \frac{1}{\sup _{(R, 1)} \mu}-\frac{1}{2 \sigma}\right) \int_{R}^{1}\left(\sqrt{\mu} v^{i}\right)^{2} d r-\frac{\beta \mu(1)}{\sqrt{k}}\left(v^{o}(1)\right)^{2} \leq \\
& -\frac{\mu(1) v^{o}(1) \omega}{2}\left(\frac{1}{\gamma^{2}}-1\right)
\end{aligned}
$$

holds. Under such a condition, each term in the left-hand side of the inequality (25) is positive. This implies the validity of estimates (19) for negative values of $\beta$. Otherwise, i.e. if $\beta \geq 0$, the positive terms in the left-hand side of (25) are estimated by two positive terms in the right-hand side. In case

$$
\frac{k}{2(\omega k+1)} \sup _{(R, 1)}>2 R^{2}
$$

i.e. if the second term in (25) is negative, estimates (25) must be rewritten as

$$
\begin{aligned}
& \left(1-\frac{\sigma}{2 R^{2}}\right) \int_{R}^{1}\left(\sqrt{\mu} \frac{d v^{i}}{d r}\right)^{2} d r \leq \\
& \left(\frac{1}{2 \sigma}-\left(\omega+\frac{1}{k}\right) \frac{1}{\sup _{(R, 1)} \mu}\right) \int_{R}^{1}\left(\sqrt{\mu} v^{i}\right)^{2} d r+ \\
& \frac{\beta \mu(1)}{\sqrt{k}}\left(v^{o}(1)\right)^{2}-\frac{\mu(1) v^{o}(1) \omega}{2}\left(\frac{1}{\gamma^{2}}-1\right)
\end{aligned}
$$

for $\sigma$ satisfying $\left(\frac{1}{2 \sigma}-\frac{k}{k \omega+1} \frac{1}{\sup _{(R, 1)} \mu}\right) \geq 0$.

Applying the weighted Friedrichs inequality (14)

$$
\int_{R}^{1} \mu\left(v^{i}\right)^{2} d r \leq\|\mu\|_{L_{1}(R, 1)}\left\|\frac{1}{\mu}\right\|_{L_{1}(R, 1)} \int_{R}^{1} \mu\left(\frac{d v^{i}}{d r}\right)^{2} d r
$$

and (26), we observe that

$$
\begin{aligned}
& \left(1-\frac{\sigma}{2 R^{2}}-\|\mu\|_{L_{1}(R, 1)}\left\|\frac{1}{\mu}\right\|_{L_{1}(R, 1)} \times\right. \\
& \left.\left(\frac{1}{2 \sigma}-\frac{k \omega+1}{k} \frac{1}{\sup _{(R, 1)} \mu}\right)\right) \times \\
& \int_{R}^{1}\left(\sqrt{\mu} \frac{d v^{i}}{d r}\right)^{2} d r \leq \\
& \frac{\beta \mu(1)}{\sqrt{k}}\left(v^{o}(1)\right)^{2}-\frac{\mu(1) v^{o}(1) \omega}{2}\left(\frac{1}{\gamma^{2}}-1\right) .
\end{aligned}
$$

Choose $\sigma$ such that the coefficient in front of the first integral in (27) be nonnegative, e.g. $\sigma$ can solve the following inequalities

$$
\left\{\begin{array}{l}
1-\frac{\sigma}{2 R^{2}}>\frac{1}{2}\|\mu\|_{L_{1}(R, 1)}\left\|\frac{1}{\mu}\right\|_{L_{1}(R, 1)} \\
0<\left(\frac{1}{2 \sigma}-\frac{k \omega+1}{k} \frac{1}{\sup _{(R, 1)} \mu}\right)<\frac{1}{2}
\end{array} .\right.
$$

By Hölder inequality

$$
\begin{aligned}
& \frac{1-R}{2}=\frac{1}{2} \int_{R}^{1} 1 d r \leq \frac{1}{2}\left(\int_{R}^{1} \mu d r\right)^{\frac{1}{2}}\left(\int_{R}^{1} \frac{1}{\mu} d r\right)^{\frac{1}{2}}= \\
& \frac{1}{2}\|\mu\|_{L_{1}(R, 1)}\left\|\frac{1}{\mu}\right\|_{L_{1}(R, 1)} \cdot
\end{aligned}
$$


Consequently,

$$
1-\frac{1}{2}\|\mu\|_{L_{1}(R, 1)}\left\|\frac{1}{\mu}\right\|_{L_{1}(R, 1)} \leq-\frac{1-R}{2}
$$

and

$$
\frac{1}{2}+\frac{k \omega+1}{k} \frac{2}{\sup _{(R, 1)} \mu}>2 R^{2}
$$

In particular, the system (28) is fulfilled for $\sigma=\frac{R^{2}}{2}$. Proceeding with this value of $\sigma$, we derive the estimate

$$
\begin{aligned}
C_{2} \int_{R}^{1}\left(\sqrt{\mu} \frac{d v^{i}}{d r}\right)^{2} d r & \leq \frac{\beta \mu(1)}{\sqrt{k}}\left(v^{o}(1)\right)^{2}- \\
& \frac{\mu(1) v^{o}(1) \omega}{2}\left(\frac{1}{\gamma^{2}}-1\right),
\end{aligned}
$$

where

$$
C_{2}=\frac{3}{4}-\|\mu\|_{L_{1}(R, 1)}\left\|\frac{1}{\mu}\right\|_{L_{1}(R, 1)}\left(\frac{1}{R^{2}}-\frac{k \omega+1}{k} \frac{1}{\sup _{(R, 1)} \mu}\right)>0
$$
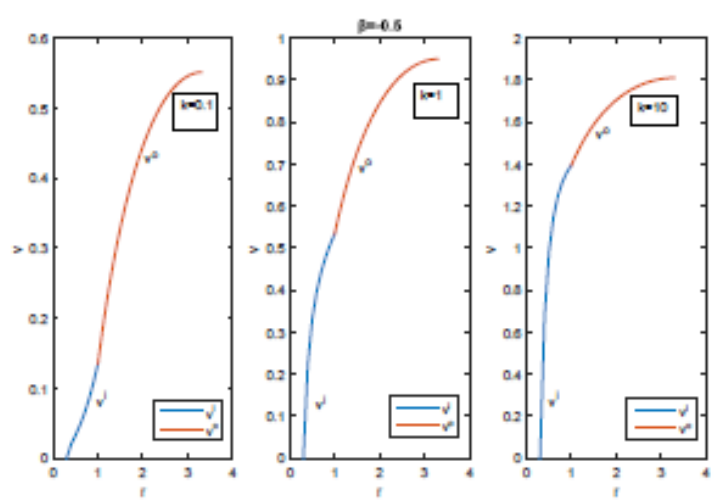

as $2 R^{2}<\frac{1}{2(\omega k+1)} k \sup \mu$. Finally, applying again the Friedrichs inequality (14), we get

$$
\begin{aligned}
& \int_{R}^{1}\left(\sqrt{\mu} v^{i}\right)^{2} d r \leq\|\mu\|_{L_{1}(R, 1)}\left\|\frac{1}{\mu}\right\|_{L_{1}(R, 1)} \int_{R}^{1}\left(\sqrt{\mu} \frac{d v^{i}}{d r}\right)^{2} d r \leq \\
& \frac{\|\mu\|_{L_{1}(R, 1)}\left\|\frac{1}{\mu}\right\|_{L_{1}(R, 1)}}{\left(\frac{3}{4}-\|\mu\|_{L_{1}(R, 1)}\left\|\frac{1}{\mu}\right\|_{L_{1}(R, 1)}\left(\frac{1}{R^{2}}-\frac{k \omega+1}{k} \frac{1}{\sup _{(R, 1)} \mu}\right)\right)} \times \\
& \left(\frac{\beta \mu(1)}{\sqrt{k}}\left(v^{o}(1)\right)^{2}-\frac{\mu(1) v^{o}(1) \omega}{2}\left(\frac{1}{\gamma^{2}}-1\right)\right) .
\end{aligned}
$$
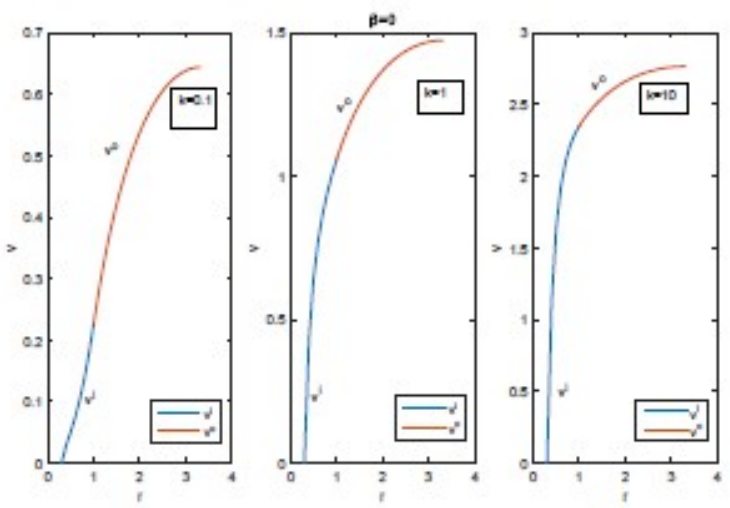

If $\beta>0$, then the left-hand side integral can be estimated by two terms in the right-hand side, while for the opposite case $\beta \leq 0$ the term $\frac{\beta \mu(1)}{\sqrt{k}}\left(v^{o}(1)\right)^{2}$ shall be moved to the left-hand side and it is not involved in the final estimate.

Estimates (19) give some restriction on growth of the solution $v^{i}$ : indeed,

$$
\left\|\sqrt{\mu} v^{i}\right\| \leq C \Leftrightarrow v^{i} \sim \frac{C}{\sqrt{\mu}} .
$$

The last relation confirms the natural phenomena: the greater viscosity $\mu$ the less the speed $v^{i}$.

One can see from (19) that for the case $\beta>0$ the velocity can take the greater values than for the opposite case ( if $\beta \leq$ $0)$.
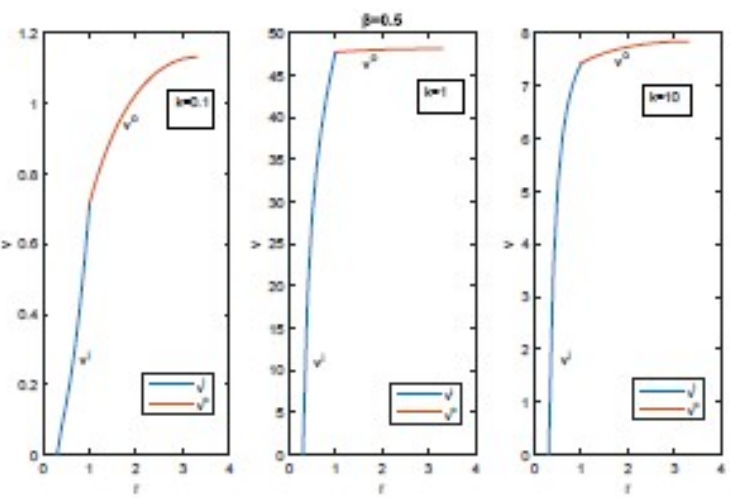

Figure 1. The behaviour of the velocity for fixed data $\gamma=0.3, R=0.3$, $\omega=-0.1$ and particular cases $\beta=-0.5,0,0.5, k=0.1,1,10$.

\section{Numerics}

Let us consider the particular case of the viscosity $\mu(r)=$ $\frac{1}{r^{2}}$. This can model variable viscosity flow decreasing in the pure liquid direction. Such situation is common for filtration through superhydrophobic surfaces [7]. In this case the weak solution $v^{i}$ can be found explicitly:

$$
v^{i}(r)=\left(\frac{C_{1}^{i}}{r^{1+\sqrt{1+\frac{1}{k}}}}+\frac{C_{2}^{i}}{r^{1-\sqrt{1+\frac{1}{k}}}}+k\right)(-\omega) .
$$


We denote for the simplicity $C_{k}=\sqrt{1+\frac{1}{k}}$. The constants $C_{1}^{i}, C_{2}^{i}$ and $C$ can be evaluated from the boundary conditions:

$$
\begin{gathered}
C_{1}^{i}=\frac{k\left(1+\frac{\beta}{\sqrt{k}}-C_{k}\right)-\frac{1}{2} R^{-1+C_{k}}\left(\frac{1}{\gamma^{2}}-1+2 \beta \sqrt{k}\right)}{R^{-1+C_{k}}\left(1+\frac{\beta}{\sqrt{k}}+C_{k}\right)-R^{-1-C_{k}}\left(1+\frac{\beta}{\sqrt{k}}-C_{k}\right)} \\
C_{2}^{i}=\frac{-k\left(\frac{\beta}{\sqrt{k}}+1+C_{k}\right)+\frac{1}{2} R^{-1-C_{k}}\left(\frac{1}{\gamma^{2}}-1+2 \beta \sqrt{k}\right)}{R^{-1+C_{k}}\left(1+C_{k}+\frac{\beta}{\sqrt{k}}\right)-R^{-1-C_{k}}\left(1-C_{k}+\frac{\beta}{\sqrt{k}}\right)} \\
C=C_{1}^{i}+C_{2}^{i}+k+\frac{1}{4} .
\end{gathered}
$$

One can see that coefficients $C_{1}^{i}, C_{2}^{i}$ and $C$ depend on initial data: $\beta, k$ as well as on the choice of bounds $R$ and $\gamma$.

The MatLab graph for velocity field is shown in Figure 1. It is seen clearly that the velocity in the porous zone takes the less values than in the pure liquid area. The positive values of parameter $\beta$ correspond to the higher velocity what agrees with theoretical results.

\section{Acknowledgments}

I thank Professor Anatoly Filippov for proposal of considered physical problem and fruitful discussions on this topic. My acknowledgments are also addressed to reviewers. Their comments and valuable remarks have improved the manuscript. The work was partially supported by RFBR (projects 17-08-01287 and 15-58-45142) and by the grant of President of Russian Federation supporting young Russian scientists (project MK-4615.2015.1)

\section{REFERENCES}

[1] J. Happel, H. Brenner, Low Reynolds Number Hydrodynamics, Martinus Nijoff, The Hague, Netherlands, 1983.

[2] Yu. M. Volfkovich, A. N. Filippov and V. S. Bagotsky, Structural properties of porous materials and powders used in different fields of science and technology, Springer-Verlag, 2014, $328 \mathrm{p}$.

[3] I. Babuska, Error-bounds for the finite element method, Numer. Math., Vol.16, (1970/1971), 322-333.
[4] H. C. Brinkman, A calculation of the viscous force exerted by a flowing fluid on a dense swarm of particles, J. Appl. Sci. Res., Vol.1, (1947), 27-34.

[5] S. Deo, A. Filippov, A. Tiwari, S. Vasin and V. Starov, Hydrodynamic permeability of aggregates of porous particles with an impermeable core, Adv Colloid Interface Sci., Vol.164, (2011), No.1-2, 21-37.

[6] P.D. Lax and A.N. Milgram, Parabolic equations, Ann. Math. Studies, Vol.33, (1954), 167-190.

[7] G. McHale, M. I. Newton and N. J. Shirtcliffe, Soft Matter, 2010, vol. 6, p. 714 .

[8] J.A. Ochoa-Tapia and S. Whitaker, Momentum transfer at the boundary between a porous medium and a homogeneous fluid- I. Theoretical development, Int. J. Heat Mass Transfer, Vol.38, (1995), 2635-2646.

[9] J. A. Ochoa-Tapia and S. Whitaker, Momentum transfer at the boundary between a porous medium and a homogeneous fluid- II. Comparison with experiment, Int. J. Heat Mass Transfer, Vol.38, (1995), 2647-2655.

[10] S. I. Vasin, Permiability of Media Composed of Impenetrable Cylinders Covered with Porous Layer, Colloid Journal, Vol.72, (2010), No. 3, 315-322.

[11] S. I. Vasin, Cell Models of Porous Media Composed of Impenetrable Spherical Particles Coated with Nonuniform Porous Layers, Colloid Journal, Vol.72, (2010), No.3, 307-314.

[12] S. I. Vasin, A. N. and Filippov, Cell Models for Flows in Concentrated Media Composed of Rigid Impermeable Cylinders Covered with a Porous Layer, Colloid Journal, Vol.71, (2009), No.2, 141-155.

[13] S. I. Vasin, E. E. Sherysheva and A. N. Filippov, Permeability of a medium consisting of cylindrical fibers with fractal porous adlayer, Colloid Journal, Vol.73, (2011), No.2, 167-175.

[14] S. Veerapaneni and M. R. Wiesner, Hydrodynamics of fractal aggregares with rapidly varying permeability, J. Colloid Interface Sci., Vol.177, (1996), No.1, 45-47.

[15] Yu. O. Koroleva, Integarl inequalities of Hardy and Friedrichs types with applications to homogenization theory, online available from http://lu.divaportal.org/smash/get/diva2:991067/FULLTEXT01.pdf 